\title{
Dimensional, similarity, and configural classification of integral and separable stimuli
}

\author{
STEPHEN HANDEL \\ University of Tennessee, Knoxville, Tennessee 37916 \\ SHIRO IMAI \\ University of Hokkaido, Hokkaido, Japan \\ and \\ PHILIP SPOTTSWOOD \\ University of Tennessee, Knoxville, Tennessee 37916
}

\begin{abstract}
Subjects classified sets of two-dimensional stimuli into two or three groups in any way they wished. For integral stimuli, classification was based on numerical balance and the similarity structure. For separable stimuli, classification was based on the dimensional structure and the configural properties of the stimulus set. These results replicate and extend those of Handel and Imai (1972). In addition, they suggest that no closed set of stimulus concepts is possible.
\end{abstract}

Recent work has reaffirmed the importance of the properties of the stimulus in such diverse tasks as identification, concept formation, free classification, and similarity judgment. One strategy has been to construct a set of multidimensional stimuli such that every stimulus would be defined by a value along each dimension. These stimuli are then used in a variety of tasks to discover associations between type of stimulus and experimental outcome. Moreover, the outcomes taken in combination can be used to converge on the nature of the properties themselves.

This approach has led to several similar concepts as a function of the experimental task. Handel and Imai (1972), following suggestions by Torgerson (1958) and Hyman and Well (1967), utilized similarity judgments and free classification to distinguish between analyzable and unanalyzable stimuli. Garner (1974) and his co-workers, as well as Lockhead and his co-workers (Monahan \& Lockhead, 1977) utilized identification and speeded classification tasks to distinguish between separable and integral stimuli. These notions are closely related: Analyzable generally corresponds to separable; unanalyzable generally corresponds to integral.

Separable or analyzable stimuli are composed of dimensions which, when combined, remain obvious, with each dimension being easy to perceive and to selectively attend to. The dimensions are perceived independently and provide the dominant organizational

Preparation of this report was supported by National Institute of Neurological and Communicative Disorders and Stroke Grant NS 14842. The authors would like to thank Dr. G. R. Lockhead for his helpful comments. Reprint requests should be sent to Stephen Handel, Department of Psychology, University of Tennessee, Knoxville, Tennessee 37916. characteristic: Similarity judgments are best represented by the city-block metric (i.e., differences along each dimension are additive), free classification is by the dimensional structure, and there are dimensional preferences in free classification (Handel \& Imai, 1972).

Integral or unanalyzable stimuli are composed of dimensions that "fuse" together, that interact so that each dimension becomes less distinct, forming a unitary whole or constructing an emergent relationship among the dimensions. The perceived stimulus may bear little resemblance to the stimulus as defined and constructed by the experimenter (see Monahan \& Lockhead, 1977, for an eloquent discussion of this point). The perceived similarity among stimuli seems to provide the dominant organizational characteristic: Similarity judgments are best represented by the euclidean metric, free classification is by the similarity structure, there are no dimensional preferences in free classification, and the similarity among stimuli predicts identification performance (Handel \& Imai, 1972; Monahan \& Lockhead, 1977).

The purpose of the present experiment was to investigate the free classification of sets of multidimensional stimuli which have a more complicated dimensional and similarity structure. Previous work, cited above, had found a clear correlation between type of stimulus and type of free classification. Separable stimuli were classified by the dimensional structure: One group would be formed for each level of one dimension so that stimuli within each group would necessarily differ on the other dimensions (e.g., red and blue chips would be classified such that one group would be all red chips, the other all blue 
chips). If stimuli varied on three or more levels of each dimension, then two-group dimensional classification would be impossible since there is no way that one level could define each group (e.g., with red, blue, and green chips, at least one group must contain chips of different colors). Integral stimuli were classified by the similarity structure: Similar stimuli would be placed in the same group and dissimilar stimuli placed in different groups. The classification would maximize the ratio of between-group dissimilarity to within-group similarity. While similarity classification is always possible, the more complex similarity structure makes this type of classification more problematic since the similarity relationships between pairs of stimuli become more varied; it should be more difficult to utilize the pairwise similarities to maximize the similarity criterion. The empirical question then was how do subjects classify these sets when dimensional classification is impossible and similarity classification is more difficult?

\section{METHOD}

\section{Subjects}

The subjects were 83 University of Tennessee undergraduates; all were tested for color vision.

\section{Stimuli}

There were five types of stimuli. Each type was two-dimensional, and each dimension had four levels.

Separable stimuli. The dimensions of the first stimuli were geometric shape and color. The shapes were circle, square, triangle, and hexagon; the colors were blue, green, yellow, and red. The levels of each dimension were nominal and the discriminability (i.e., dissimilarity among levels) was high. The stimuli were made from colored construction paper. All forms were equated in subjective size based on preliminary experimentation (average area was $4.5 \mathrm{~cm}^{2}$ ) and were mounted on white matte cardboard backing measuring $5 \times 9 \mathrm{~cm}$.

The dimensions of the second set of stimuli were size of square and achromatic Munsell lightness. The discriminability among levels of each dimension was low. The levels of the size dimension in terms of the length of one side were $1.5,1.9,2.3$, and $2.7 \mathrm{~cm}$. The levels of the lightness dimension were N3, N4, N5, and N6. The squares were cut from glossy Munsell paper, but not mounted.

The dimensions of the third set of stimuli were also size of square and achromatic Munsell lightness. In contrast with the second set, the discriminability between levels of each dimension was high. The levels of the size dimension in terms of the length of one side were $.3,1.5,2.7$, and $4.0 \mathrm{~cm}$. The levels of the lightness dimension were $\mathrm{N} 1, \mathrm{~N} 3.5, \mathrm{~N} 6$, and $\mathrm{N} 8.5$.

Integral stimuli. The dimensions of the first set of integral stimuli were Munsell value and chroma: All chips were the same hue-5RP. The levels of the value dimension were $/ 3 /, / 4 /, / 5 /$, and $/ 6 /$. The levels of the chroma dimension were $4,6,8$, and 10 . The chips were glossy $(2.5 \times 3.7 \mathrm{~cm})$ and mounted on white matte cardboard backing $(5 \times 9 \mathrm{~cm})$.

The above four sets of stimuli were identical to those used previously (Handel \& Imai, 1972). A schematic for each set and the judged similarities between each pair of stimuli are given in that paper.

The dimensions of the second set of integral stimuli were more complicated. The first was a triangle shape. Imagine a right triangle, with the right angle forming an "L." Now, if the upper vertex is successively displaced to the left, it will generate triangles in which the right angle gets progressively more obtuse. Note that the area is constant, as the base and altitude do not vary. The base of the triangles was $3 \mathrm{~cm}$, and the altitude was $4.5 \mathrm{~cm}$. The four levels of this dimension occurred when the right angle increased to $99 \mathrm{deg}$ (.4-cm displacement), $108 \mathrm{deg}$ (1.2-cm displacement), $117 \mathrm{deg}$ (2.2-cm displacement), and $126 \mathrm{deg}$ (3.2-cm displacement). The triangle was filled in by a horizontal band of candy-apple red along the base adjacent to a horizontal band of candy-apple green reaching the apex. The four levels of this dimension were constructed by increasing the height of the red band, thereby decreasing the height of the green band. The four levels, with the height of the red band being measured from the base, were $1,1.5,2.1$, and $2.7 \mathrm{~cm}$. $^{1}$

\section{Stimulus Sets}

Each dimension pair may be represented by a 4 by 4 matrix, with the rows and columns representing the four levels of each dimension. All stimulus sets contained four stimuli and, therefore, may be portrayed by four dots in the appropriate cells symbolizing the stimuli. To achieve sets in which two-group dimensional classification was impossible, only those that placed stimuli in a 3 by 3 or a 3 by 4 submatrix were used.

Many possible sets are equivalent in that the dimensional and similarity structures among the stimuli are identical although the actual stimuli differ. This equivalence may be understood by imagining the dots as a visual pattern. Equivalent sets can be produced by translating, reflecting, and/or rotating by 90 or $270 \mathrm{deg}$ the dot pattern within the entire 4 by 4 matrix. In addition, for the geometric shape $\times$ color stimuli, sets are equivalent if they can be produced by interchanging the nominal levels of the shape and color dimensions.

On the basis of the logical structure, all the possible stimulus sets were partitioned into a smaller number of unique sets. There were 10 different sets that fit into a 3 by 3 matrix and 9 sets that fit into a 3 by 4 matrix. However, logically equivalent sets may not be classified identically. This suggests an experimental procedure. For each unique set, present at least two equivalents to determine if each is classified identically; if not, present other equivalents of the same unique set to obtain reliable estimates of the percentages of differing classifications.

There was a total of 23 sets of four stimuli: the 19 unique sets possible within a 3 by 3 or a 3 by 4 matrix plus 4 sets within a 2 by 4 matrix used to compare these results with those of Handel and Imai (1972). No differences were found, and these latter sets will not be discussed further.

\section{Procedure}

The experimenter faced the subject across a table and placed the stimuli in front of the subject in a random arrangement. The subjects were free to place the stimuli into two or three groups in any way they wished. Following this, each subject was required to classify the same stimuli into the alternative number of groups, two or three.

\section{Experimental Design}

There were four experimental segments. In the first, two equivalents of every unique set for the size $\times$ lightness, Munsell value $\times$ chroma, and geometric shape $\times$ color sets were presented. The two equivalents were chosen to reverse the dimensional structure; if the column dimension was represented by four levels in one equivalent, the row dimension was represented by four levels in the other equivalent. There were 150 sets in all. These were broken into two sections, and nine subjects classified each section on successive days.

The second segment was designed to generalize the results from the first segment. It further sampled equivalents of those sets in which the preferred two-groups classification was ambiguous. Across the four dimension pairs listed immediately above, there was a total of 82 sets. These sets were split in half, and nine different subjects classified each group of 41 sets.

The third segment further explicated the results. In this segment, the subjects classified only one dimension pair to determine if 
alternating between types of dimensions as in the first two segments affected classification. For the Munsell value $\times$ chroma sets, four equivalents for each of six sets ( 24 in total) were presented to eight subjects. For the low-discriminability size $\times$ lightness sets, four equivalents for each of 12 sets (48 in total) were presented to eight different subjects. For the high-discriminability size $\times$ lightness sets, there were four equivalents for each of 15 sets (60 in total). These were split equally into two groups, and each group of 30 was presented to eight different subjects. Thus, there was a total of 32 subjects.

In the fourth segment, the triangle $x$ color ratio sets were presented alone. There were 46 sets, two equivalents for each of the 23 sets. All sets were presented to 24 new subjects.

For all segments, each unique set was always represented by an even number of equivalents, thereby balancing the dimensional and similarity siructure. Furthermore, for the first two segments, sets from different dimension pairs were alternated, and for all segments, the order of presentation was counterbalanced across subjects.

\section{RESULTS}

Preliminary analyses indicated that the various experimental segments produced similar results, so the results were combined. In what follows, only two-group classification will be discussed extensively. The results from three-group classification show the identical trends, and these will be used only to supplement the results from two-group classifications.

\section{Integral Stimuli}

The free classification of both types of integral stimuli-Munsell value $\times$ chroma and triangle $\times$ color ratio-was based on numerical balance and perceived similarity. Subjects preferred balanced classifications with two stimuli in each of two groups, such that the stimuli within each group were maximally similar and those in different groups were maximally dissimilar. There was no effect of the dimensional structure. Representative stimulus sets chosen to illustrate the interplay between similarity and balance for sets within $3 \times 3$ and $3 \times 4$ matrices are shown in Figure 1.

The four stimuli in a set are represented by their positions within the $4 \times 4$ matrix. For any classification, the stimuli in one group are portrayed by $\times s$, while the stimuli in the second group are portrayed by $s$. The percentage choice of each such classification is listed below. For each set, the two optimal similarity classifications are shown. ${ }^{2}$ Thus, for Set 12 , the best similarity classification was a balanced 2-2 classification, and it was chosen $60 \%$ and $67 \%$ of the time for the Munsell value $\times$ chroma and triangle $\times$ color ratio sets, respectively. The second best similarity classification was an unbalanced 3-1 classification, and it was chosen $3 \%$ and $8 \%$ of the time for the Munsell value $\times$ chroma and triangle $\times$ color ratio sets, respectively. For all sets, the preferred classification was one of these two best similarity classifications. It should be noted that for two sets $(11,17)$, there are several logically identical ways of

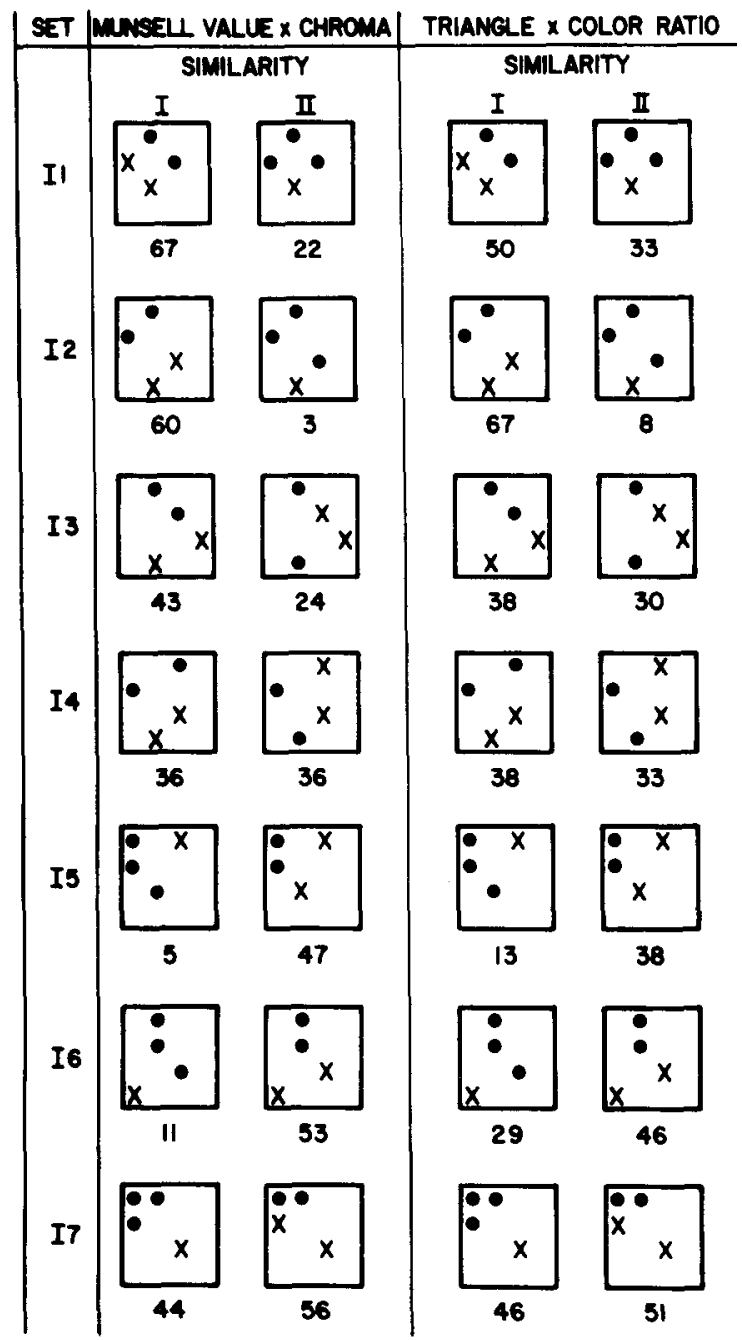

Figure 1. Integral stimuli: the percentage of use of the two optimal similarity classifications for the Munsell value $\times$ chroma and triangle $\times$ color ratio stimuli.

achieving the same similarity classification. For example, there are four identical instances of the second classification of Set I1 achievable by isolating one of the four stimuli. In these cases, the percentage reported is the sum across the possibilities.

Averaging across all sets, if the optimal similarity classification was balanced (Sets I1-I4 in Figure 1), this classification was preferred. For the Munsell value $x$ chroma sets, it was chosen $58 \%$ of the time; for the triangle $\times$ color ratio sets, it was chosen $59 \%$ of the time. In contrast, if the optimal similarity classification was unbalanced (Sets 15-17), it was not preferred. The preferred grouping was balanced and it was usually the second best similarity classification. For the Munsell value $\times$ chroma sets and for the triangle $\times$ color ratios sets, the unbalanced, optimal similarity classification was chosen $21 \%$ and $28 \%$ of the time, respectively, while the balanced, similarity classification was chosen $46 \%$ and $49 \%$ of the time, respectively. The highest percentage of optimal 
similarity, unbalanced classifications occurred for Set I7. In this case, the three stimuli within a group represent the maximum attainable within-group similarity. Thus, while similarity can act to moderate the preference for numerical balance, it still functioned within the primary constraint imposed by numerical balance.

\section{Separable Stimuli}

The classification of all types of separable stimuli was based on the dimensional structure. While strict dimensional classification was impossible, subjects maximized the "dimensionalness" of a classification by grouping pairs of stimuli that differed on only one dimension or by producing a classification that emphasized the structure along one dimension. In what follows, stimulus sets within a $3 \times 3$ matrix will be used to assess the preference for classification using the row as opposed to the column dimension. Stimulus sets within a $3 \times 4$ matrix will be used to assess the preference for classifications that grouped stimuli differing on one dimension as opposed to those that emphasized the configural nature of the dimensional structure. In both instances, similarity affected only to a small degree the percentage choice of the various classifications based on the dimensions.

Dimensional preference: $3 \times 3$ matrix. When four stimuli are placed in a $3 \times 3$ matrix, one pair of stimuli differs on the row dimensions and one pair differs on the column dimension. Thus, it is possible to maximize the "dimensionalness" of a classification by placing one of these pairs in a group and pairing the remaining two stimuli. The remaining two stimuli would necessarily differ on both dimensions. These sets, therefore, can be used to study dimensional choice (i.e., which pair is placed in a group) as a function of the similarity structure. Overall, there was a preference for lightness classification for the size $\times$ lightness stimuli. Similarity affected only the strength of this preference. There were no preferences for the geometric shape $\times$ color stimuli.

Representative sets for the size $\times$ lightness stimuli are shown in Figure 2. For both low- and highdiscriminability sets, the percentage of lightness classification grouping the two stimuli identical in lightness and the percentage of size classification grouping the two stimuli identical in size are shown for a set and its equivalent.

There are three kinds of sets that vary the similarity relationships among the two stimuli differing only in lightness or size. For the first three sets (S1-S3), the similarity between the pairs differing on one dimension was equal. That is to say, the dissimilarity in size for two stimuli of the same lightness equals the dissimilarity in lightness for two stimuli of the same size. In addition, classification with either dimension results in an equally dissimilar pair in the second group. There is no rationale for either light-

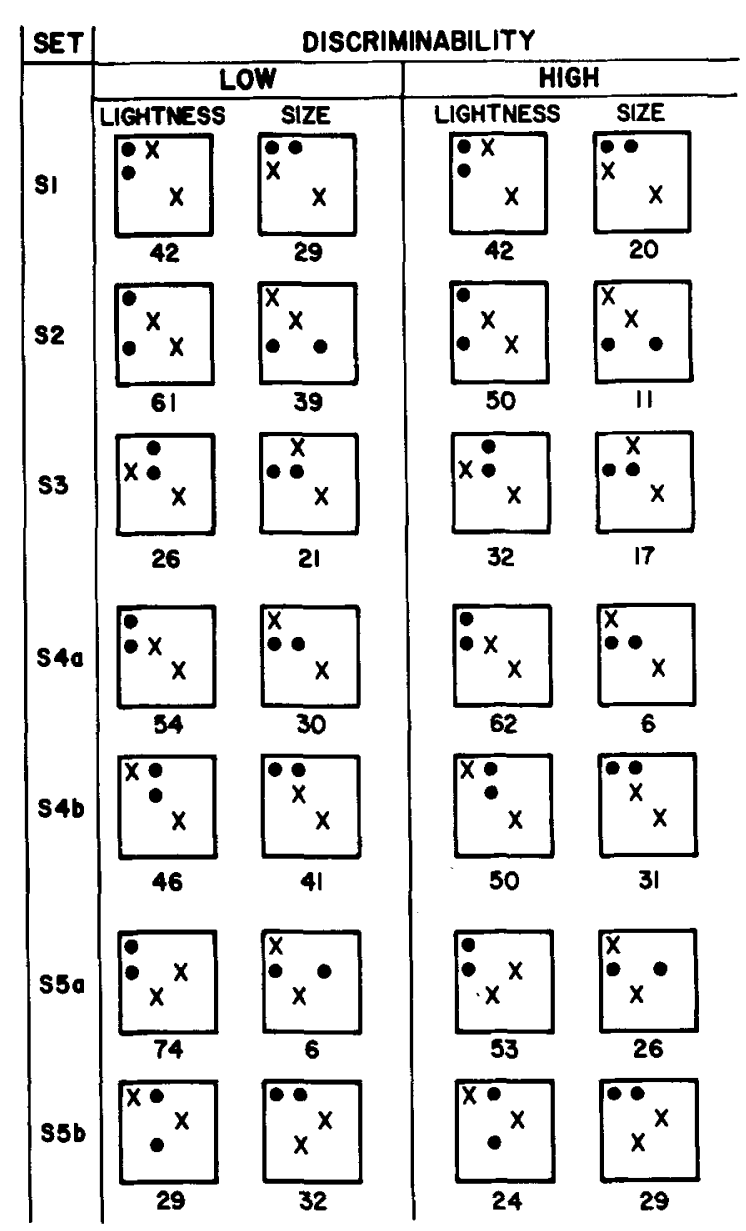

Figure 2. Separable stimuli: the percentage of lightness and size classifications for the low- and high-discriminability size $\times$ lightness stimuli.

ness or size classification on either dimensional and/ or similarity grounds; classification must be based on preference. Here, lightness classification predominated: $42 \%$ of the classification used the lightness dimension and $23 \%$ used the size dimension.

For the fourth set (S4), the similarity between stimuli differing on one dimension was again identical. However, classification by size or lightness determined the similarity within the second group, the dissimilarity between the groups, and the nature of the classifications. Consider Set S4a. Lightness classification (i.e., grouping the two stimuli with the same level on the lightness dimension) as contrasted with size classification resulted in (1) the two stimuli paired in the second group being more similar, (2) the two subgroups being more dissimilar, and (3) an ordinal classification in which lightness of both stimuli in the second group was greater than that of the stimuli in the first group. The reverse was true for Set S4b: Size classification would be optimal on all three criteria. Empirically, the percentage of lightness classification increased in S4a and the percentage of size classification increased in S4b. 
Finally, Set S5 introduced yet another complexitythe similarities between the two stimuli identical in lightness and those identical in size differed. For S5a, the dissimilarity in size for stimuli of the same lightness was smaller than the dissimilarity in lightness for stimuli of the same size. On this basis, lightness classification should be selected. Moreover, lightness rather than size classification optimized the three criteria listed above-high within-group similarity, high between-group dissimilarity, and ordinal grouping. All of these factors should reinforce the general tendency for lightness classification, and this was clearly true for the low-discriminability set, although less so for the high-discriminability set. For S5b, size classification was optimal according to all these criteria and all should act to counter the overall predilection for lightness classification. This, in fact, was the outcome: Only for Set S5b was size classification predominant.

The classification of the geometric shape $\times$ color stimuli was quite straightforward: $39 \%$ of the classifications were by the shape dimension and $39 \%$ were by the color dimension. There was no dominant dimension.

Dimensional preference and configural classification: $3 \times 4$ matrix. When four stimuli are placed in a $3 \times 4$ matrix, one pair of stimuli differ on one dimension, but the remaining two stimuli differ from each other and from the above two on both dimensions. The possible classifications differ from sets in a $3 \times 4$ matrix since there is only one way in which stimuli differing on a single dimension can be paired.

For those sets in which lightness classification (i.e., grouping into two stimuli of identical lightness) was possible (" $a$ " sets), the preferred classifications were a balanced lightness classification and an unbalanced lightness classification grouping the three lightest or three darkest stimuli. For those sets in which size classification (i.e., grouping two stimuli of the identical size) was possible ("b" sets), the preferred classifications were based on the lightness dimension. Two classifications were utilized: (a) for the low discriminability size $\times$ lightness stimuli, the four stimuli were split such that the lighter grays were placed in one group and the darker grays in the other; or (b) for the high discriminability size $\times$ lightness stimuli, the four stimuli were split such that the lightest and darkest grays were placed in one group and the middle grays in the other.

Representative sets are shown in Figure 3. For each set, the "a" equivalent contains two stimuli of the same lightness, and the percentages of balanced lightness and unbalanced lightness classifications are shown. The " $b$ " equivalent contains two stimuli of the same size, and the percentages of lighter/darker and end/middle lightness classifications are shown.

Consider first those equivalents in which lightness classification (pairing the two stimuli identical in

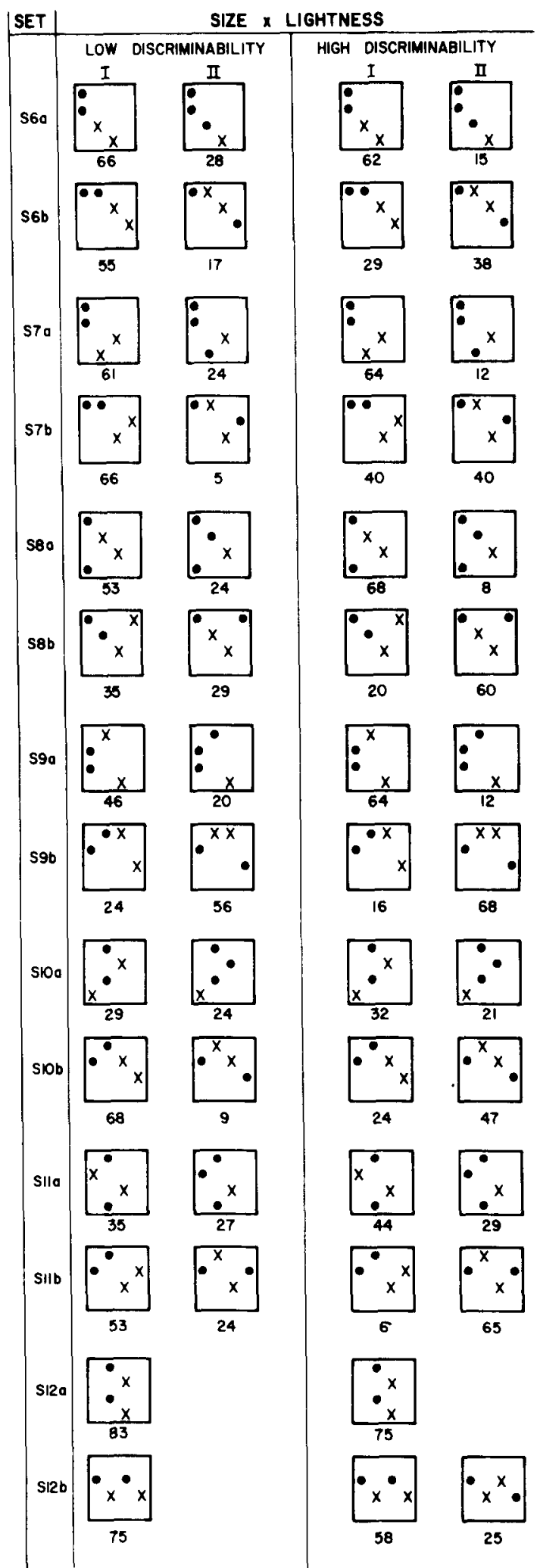

Figure 3. Separable stimuli: the classification of low- and highdiscriminability size $\times$ lightness stimuli. For the " $a$ " equivalent, the percentages of balanced and unbalanced lightness classifications are shown. For the " $b$ " equivalent, the percentages of lighter/ darker lightness classifications and ends/middle lightness classifications are shown. 
lightness) was possible (S6a-S11a). For both the highand low-discriminability sets, lightness classification predominated. The percentage of lightness classification was maximum $(61 \%)$ when the identical stimuli were at the end of the continuum (Sets S6a-S9a). The percentage was lowest $(39 \%)$ when the level of lightness at which the stimuli were identical was in the middle of the continuum (e.g., S10a, S11a). To group the two identical lightness stimuli, the remaining two stimuli that bound the middle lightness level must be paired. This classification violates the ordinal properties of the lightness dimension and was used less frequently. An unbalanced 3-1 lightness classification generating a lighter vs. darker split thereby gained in relative use. The magnitude of the size dissimilarity between the two identical lightness stimuli did not significantly affect the percentage of lightness classification.

Now consider those sets in which size classification was possible (Sets S6b-S11b). Two predominant types of classification were found, both based on lightness and both violating the size dimension structure. In the first type, the lightness dimension was split up the middle so that one group contained the darker grays, while the second group contained the lighter grays. The classification maximized the similarity within groups and dissimilarity between groups based on the lightness dimension. This outcome is reminiscent of similarity based on the dominance metric (Hyman \& Well, 1967) in which subjects utilize only one dimension out of many. In the second type, the lightness dimension was split, ends vs. middles: One group contained the whitest and blackest, while the other contained the intermediate grays. It may be conceptualized as a matching or balancing classification.

The low discriminability size $\times$ lightness sets were classified by the former strategy: lighter grays against the darker grays. Overall, $55 \%$ of the classifications split the lightness dimension up the middle, while $20 \%$ of the classifications grouped the extreme lightness stimuli against the middle lightness stimuli. Sets S8b-S1 1b generated the strongest test: To classify lighter grays against darker grays, the two equal-size stimuli must be split apart, and even here, lighter/ darker grouping was chosen $(45 \%)$. For only one set (S9b) was lighter/darker not the preferred classification.

The high-discriminability size $\times$ lightness sets were classified by the latter strategy: white and black against the middle grays. Overall, $54 \%$ of the classifications were of this type, while $22 \%$ split the lightness dimension into lighter vs. darker groups. There are two cases. In the first (S5b, S7b, S10b), to achieve end/ middle classification, the two identical size stimuli must be split; in this case, $42 \%$ of the classifications were of this sort. In the second (S8b, S9b, S11b), end/middle classification is simultaneously a size dimensional classification; in this case, $66 \%$ of the classifications were of this sort.

Three-group classification illustrates the same difference between the low- and high-discriminability sets. Consider S10b: Three-group size classification would occur if two equal size stimuli (in row 2) were paired, lighter/darker lightness classification would occur if either the two lightest or darkest stimuli were paired, and an end/middle lightness classification would occur if the two end or two middle stimuli were paired. For the low- and high-discriminability sets, $34 \%$ and $27 \%$ were size classifications, $31 \%$ and $9 \%$ were lighter/darker lightness classifications, and $25 \%$ and $43 \%$ were end/middle lightness classifications, respectively.

It is clear, therefore, that stimulus sets which vary across four levels of the lightness dimension can be used to distinguish between the low- and highdiscriminability sets. For the low-discriminability sets, in which all levels are grayish, the classification was based on lightness similarity. For the highdiscriminability sets, in which the levels range from white to black, classification was based on more configural properties: The groups are balanced along the lightness dimension.

The classification of the geometric shape $\times$ color sets was again straightforward. As found previously, there were no distinct dimensional preferences, and the dimensional structure determined classification. When color classification was possible, $48 \%$ of the classifications were of this type; when shape classification was possible, $59 \%$ were of this type.

\section{DISCUSSION}

The results replicate those of Handel and Imai (1972). Integral stimuli were classified by the similarity structure; separable stimuli were classified by the dimensional structure, either by grouping stimuli that differed on only one dimension or by grouping stimuli on the basis of alternate relationships along one dimension.

Handel and Imai (1972) argued that integral stimuli were classified by the similarity structure. For this reason, classification would not change either as the dimensional relationships among stimuli change or as new stimuli are added to a set. Similarity would still provide a viable basis for classification. This prediction was confirmed in the present experiment and generalized by the use of the triangle $x$ color ratio.

Why were the triangle $x$ color ratio stimuli perceived integrally? In contrast with the Munsell value $x$ chroma stimuli in which the two dimensions appear to fuse producing a new complex dimension, the triangle and color ratio dimensions appear to maintain their separate identities. Supporting this view were informal tests in which subjects could sort 
the stimuli dimensionally, either by triangle orientation or by color ratio. Monahan and Lockhead (1977) suggest one alternative. If two dimensions combine to yield a relationship connecting them, then stimuli formed by these two dimensions will be perceived integrally. A second alternative, suggested by Kingsbury (1968), is that perception shifts toward integrality as stimuli get more complex: Subjects become overwhelmed by the stimultaneous variation of many dimensions and resort to direct perceptual judgments. It may be, however, that the extra dimensions merely admit the possibility for perceptual relationships to occur, and that it is not complexity per se. In the present experiment, this possibility would argue that the stimuli generated by the two defined dimensions were not perceived as such by the subjects. Variation of the color ratio and triangle dimensions may have been perceived as variation in "uprightness," "thinness," "'apparent area," "color balance," and so on. Note the difficulty in a prior specification: It seems a matter of chance when the experimenter's generating rules match the subject's perception.

Handel and Imai (1972) argued that separable stimuli were classified by the dimensional structure. For this reason, classification should change as the dimensional structure is varied; indeed, this was the outcome. The manner in which the classification changed was a function of the dimensional structure of the stimulus set itself as well as of the discriminability of the lightness dimension.

Two cases can be distinguished. In the first case, two stimuli were the same lightness level (S1-S5, S6aS11a). For both low- and high-discriminability sets, these two stimuli were invariably grouped together, with the two remaining stimuli paired. The choice of this classification reflected an insensitivity to the difference in size between the two lightness stimuli or to the similarity among stimuli along the second dimension. What did affect the classification was the distribution along the lightness dimension. If the two stimuli identical in lightness were in the middle so that a lighter and darker stimuli were paired in the "remainder" group, then this classification was less preferred. The lightness classification appears therefore to be motivated by two factors: (1) the placement of identical stimuli in one group, and (2) the simultaneous creation of an ordinal split along that dimension.

In the second case, the four stimuli varied across the four levels of the lightness dimension and only size classification was possible (Sets S6b-S11b). For the low-discriminability sets, classification utilized similarity along only the lightness dimension. The lighter shades were placed in one group and the darker shades were placed in the second group. The two-dimensional structure was collapsed into a onedimensional structure and classification then was based on the similarities among the levels of that dimension. In contrast, while the dimensional structure also was collapsed into the lightness dimension for the high-discriminability sets, the similarity among stimuli along that dimension did not predict classification. Rather, the extremes-white and black-were grouped against the intermediate grays.

There are several possible factors contributing to this classification. By categorizing the white and black against grays, the two groups have the same average lightness. The unit of analysis has shifted from the stimulus to the group; the groups themselves are now similar in composition, at the expense of placing dissimilar stimuli in one group. Moreover, white and black are end anchors. In the sense that a horizontal and a vertical line are more similar to each other than either is to an intermediate angle, so too would white and black be more similar to each other than to gray. On top of this, white and black are verbal associates. These factors are not mutually exclusive and probably all influenced the choice of classification.

It is important to note that end/middle grouping will not be the dominant classification if dimensional classification by size is possible. This is shown in Figure 3 by sets S12a-b ${ }^{3}$. Here there are four levels of lightness, but dimensional classification by size is the preferred grouping. In addition, white and black stimuli will not be grouped together when the stimuli differ only on the achromatic lightness dimension. The sets-(a) N1, N4, N5, N8, and (b) N1, N3, N6, $\mathrm{N} 8$ - are classified up the middle into lighter against darker $(41 \%)$ or by an unbalanced 3-1 split in which the white or black form a separate groups $(25 \%)$. Only $18 \%$ of the classifications were end/middle (see Imai \& Handel, 1971).

What all this means is that the possibilities for classifications are emergent and depend on the relationships among the entire set of stimuli. Moreover, these possibilities cannot be foreseen from the classification of unidimensional stimuli or the classification of multidimensional stimuli with a restricted set of interrelations. The distinction between separable and integral captures but a part of the differences among types of stimuli. There are the differences in classification among separable stimuli found here, and we believe that similar differences would exist for integral stimuli given the right situation. This view would argue that the specific results (i.e., the effects of the endpoints of the lightness dimension) merely represent configural effects attributable to these stimuli, but in no way exhaust the possible types of configural classifications. A stimulus can be perceived and encoded in many ways, depending on the observer's predisposition, set, motivation, development, training, experience, and so on, as well as on the task requirements and constraints (see Nickerson, 1978, for a similar view). An exhaustive listing of the varieties of configural 
classification may be impossible, suggesting that an exhaustive categorization of dimensional relationships and interactions may also be impossible.

\section{REFERENCES}

Garner, W. R. The processing of information and structure. Potomac, Md: Erlbaum, 1974.

HANDEL, S., \& ImAI, S. The free classification of analyzable and unanalyzable stimuli. Perception \& Psychophysics, 1972, 12, 108-116.

Hyman, R., \& Well, A. Judgments of similarity and spatial models. Perception \& Psychophysics, 1967, 2, 233-248.

IMAI, S., \& HANDEL, S. Hierarchical stimulus and preference structures in the classification of one-dimensional stimuli. Japanese Psychological Research, 1971, 13, 192-205.

KInGSBURy, N. R. The context effect in multidimensional scaling. Unpublished doctoral dissertation, Johns Hopkins University, 1968.

Monahan, J. S., \& Lockhead, G. R. Identification of integral stimuli. Journal of Experimental Psychology: General, 1977, 106, 94-110.

Nickerson, R. S. Comment on W. R. Garner's "Selective attention to attributes and to stimuli." Journal of Experimental Psychology: General, 1978, 107, 452-456.

Torgerson, W. S. Theory and methods of scaling. New York: Wiley, 1958.

\section{NOTES}

1. On the surface, these stimuli would appear to be separable. It is easy to imagine varying the two dimensions independently, yet preliminary research using similarity judgments indicated that the stimuli were integral, best fitted by the euclidean metric.

The similarity judgment experiment was simple and straightforward. Eight naive subjects judged the similarity between each of the 120 stimulus pairs. The subjects rated similarity on a 10-point scale, with 1 representing a very similar pair and 10 representing a very different pair. The number 0 was said to represent zero difference, and subjects were asked to use the numbers so as to represent the ratios of the dissimilarities. Subjects were pretrained with 10 pairs, and the order of presentation was counterbalanced across subjects.

Preliminary analyses indicated that the dimensions were independent: The differences between two levels of one dimension did not vary as a function of the second dimension. Moreover, the differences between levels of each dimension were equated. For example, the differences between pairs of adjacent triangles ( 99 vs. $108 \mathrm{deg} ; 108 \mathrm{vs} .117 \mathrm{deg} ; 117 \mathrm{vs} .126 \mathrm{deg}$ ) were equal, as were the differences across two levels of the dimension ( 99 vs. $117 \mathrm{deg}$; $108 \mathrm{vs} .128 \mathrm{deg}$ ). On the basis of these outcomes, the 120 stimulus pairs were organized into 15 logically different pairs which represent a single type of difference. The average similarity for each logical pair was calculated separately for each subject.

To determine which distance metric was most appropriate, it was assumed that the similarity judgments of the one-dimensional differences were accurate and these were used to predict the twodimensional differences. Distance exponents for Minkowski metrics ranging from 1 to 2-the exponent for the city-block metric being 1 and the exponent for the euclidean metric being 2-were used to predict the two-dimensional differences. The measure of fit was the sum of the absolute deviations between the actual and predicted two-dimensional similarities.

The best fitting metrics for each of the eight subjects were $1.8,1.6,1.9,1.5,1.8,1.6,1.2$, and $1.4(\bar{X}=1.6)$. If the similarity judgments were first averaged across subjects, then the best fitting metric was 1.55 , closely approximating the above. Five subjects produced judgments close to the euclidean metric ( 1.6 or greater), two were intermediate $(1.5,1.4)$, and one produced judgments close to the city-block metric. For comparison purposes, in previous work, the exponents for the integral Munsell stimuli and the separable size $\times$ lightness stimuli were 1.7 and 1.2 , respectively (Handel \& Imai, 1972).

In sum then, the similarity judgments suggested that these stimuli were perceived integrally. However, there seem to be more individual differences than found for the Munsell value $\times$ chroma stimuli.

2. The similarity criterion was the maximum ratio of averaged between-group sinnilarity divided by average within-group similarity. For sets of four stimuli, there were six similarities among the four stimuli. For a 2-2 classification, two similarities were within-groups and four similarities were between-groups; for a 3-1 classification, three similarities were within-groups and three were betweengroups (i.e., a group of one stimulus is not considered to have within-group similarity). The similarity ratio was calculated using (a) the actual judged similarities from Handel and Imai (1972) for the Munsell value $\times$ chroma and size $\times$ lightness stimuli and from the present experiment (see Footnote 1) for the triangle $x$ color ratio stimuli, (b) idealized similarities based on the euclidean metric, and (c) idealized similarities based on the city-block metric. All three procedures yielded the same two best classifications.

3. These data were collected by Handel and Imai (1972) but not reported in that publication.

(Received for publication April 3, 1980; revision accepted June 17,1980 .) 